\title{
Plexiform vascular structures in the human digital dermal layer: A SEM-corrosion casting morphological study
}

\author{
A. MANELLI ${ }^{1}$, S. SANGIORGI ${ }^{2}$, M. RONGA ${ }^{3}$, M. REGUZZONI ${ }^{1}$, A. BINI ${ }^{3}, \&$ \\ M. RASPANTI ${ }^{1}$
}

Departments of ${ }^{1}$ Human Morphology, Laboratory of Human Morphology, ${ }^{2}$ Surgical Sciences - Neurosurgical Unit, and

${ }^{3}$ Department of Orthopaedics and Traumatology, University of Insubria, Via Monte Generoso, 71, 21100 Varese, Italy

\begin{abstract}
This study aimed to describe the impressive diversity of vascular plexiform structures of the hypodermal layer of human skin. We chose the human body site with the highest concentration of dermal corpuscles, the human digit, and processed it with the corrosion casting technique and scanning electron microscopy analysis (SEM). This approach proved to be the best tool to study these microvascular architectures, free from any interference by surrounding tissues. We took high-definition pictures of the vascular network of sweat glands, thermoreceptorial and tactile corpuscles, the vessels constituting the glomic bodies and those feeding the hair follicles. We observed that the three-dimensional disposition of these vessels strictly depends on the shape of the corpuscles supplied. We could see the tubular vascularization of the excretory duct of sweat glands and the ovoid one feeding their bodies, sometimes made up of two lobes. In some cases, knowledge of these morphological data regarding the normal disposition in space and intrinsic vascularization structure of the dermal corpuscles can help to explain many of the physiopathological changes occurring during chronic microangiopathic diseases.
\end{abstract}

Keywords: Human digit, corrosion casting technique, scanning electron microscope

\section{Introduction}

The human skin is an organ with multiple functions (heat regulatory, secretory, immunological, mechanical, sensorial) mostly driven to maintain and modify the relationships existing between the body and its external environment.

To accomplish this task, the skin must rely on many specialized microscopic structures located just beneath the corneal stratum in the dermal layer: sweat glands, thermoreceptorial and tactile corpuscles, glomic bodies and hair follicles (Edwards 1960; Roth 1967; Straile 1969; Backhouse 1981; Bryce \& Chizuka 1988).

This study focused on their vascular support imaging the three-dimensional architecture of their microvessels by corrosion casting and scanning electron microscopy.

This technique proved the best method to investigate the vascular network of the deeper skin layers disclosing not only different structural conformations but also subtle ultrastructural details (Murakami 1971; Hodde \& Nowell 1980; Weiger et al. 1986; Castenholz 1989; Lametschwandtner et al. 1990).
From a three-dimensional point of view, little is known about the vascular support of the dermal corpuscle (Pollitt \& Molyneux 1990; Yamamoto 1990; Sakita et al. 1994; Nasu et al. 1998). In addition, knowledge of the actual disposition of these structures in space and their morphological features can help to explain the changes occurring during many pathophysiological conditions such as thermoregulatory impairment and collagenic diseases (vasculitis, microangiopathies and Raynaud's syndrome) (Grant \& Bland 1930; De Takats 1932; Conrad 1971).

For all these reasons, this study aimed to make a detailed description of the microvascular structures feeding dermal corpuscles, building up a map of their shape and position in space.

\section{Materials and methods}

We injected a low viscosity resin (MERCOX) into a human digit traumatically explanted from the hand of a 56-year-old man. The entity of the trauma precluded reimplantation of the digit and all the procedures were undertaken in accordance with the

Correspondence: A. Manelli, Department of Human Morphology, Laboratory of Human Morphology, University of Insubria, Via Monte Generoso, 71, 21100 Varese, Italy. Tel: +39-332-264847. Fax: +39-332-263324. E-mail: emanelli@libero.it 
ethical guidelines approved by the University of Insubria.

The digit was maintained at $4{ }^{\circ} \mathrm{C}$ before the injection. The main digital artery was exposed under a dissection stereomicroscope (Leica - WILD M3C) and shortly after a $24 \mathrm{G}$ cannule was inserted and tied to the vessel with silk ligature. This device was then connected to a three-way system previously filled with saline solution.

The injecting procedure started with a first infusion of $10 \mathrm{ml}$ heparinized solution $0.3 \%$ to prevent blood from clotting and wash away all red blood cells. The pressure of injection was monitored manually paying attention not to cause any interstitial oedema (empirical evidence was the swelling of digital soft tissues). A second injection of $5 \mathrm{ml}$ of saline solution was necessary to remove all the blood and to wash out the heparin from the vascular bed.

We proceeded with fixation of the vascular bed with $5 \mathrm{ml}$ Karnovsky solution $(0.25 \%$ glutaraldehyde and $0.25 \%$ paraformaldehyde in $0.1 \mathrm{M} \mathrm{Na-cacody-}$ late buffer at $\mathrm{pH}$ 7.2) to prevent the resin leaking out of the vascular bed and to reduce the modifications occurring in the endothelial cells. The resin $(5 \mathrm{ml}$ MERCOX and $0.2 \mathrm{ml}$ benzoyl peroxide) was then injected through the cannule until the reflux from the venous vessel became evident and the viscosity of the resin itself appeared slightly increased due to the beginning of the hardening processes.

The finger was then immersed in a warm water bath $\left(60^{\circ} \mathrm{C}\right)$ to leave the resin to harden for about $2 \mathrm{~h}$.

The digestion process consisted several baths of a solution of potassium hydroxide $(15 \%)$ changed every $12 \mathrm{~h}$ at room temperature for 5 days. Decalcification was obtained using hydrochloric acid (3\%).

The cast, cleared from tissues, was dissected under a dissection microscope (WILD M3C - Leica) before the SEM preparation procedure.

The resulting casts were rinsed in distilled water (to prevent deposition of $\mathrm{KOH}$ or $\mathrm{HCl}$ salt), dehydrated in graded alcohol, critical point dried in an Emitech K850 CPD apparatus and mounted by silver paste and metallic bridges (Lametschwandtner et al. 1980) on aluminium stubs.

The mounted specimens were then coated with $10 \mathrm{~nm}$ gold in an Emitech K250 sputter-coater and observed with a Philips XL-30 FEG scanning electron microscope operating at $10-15 \mathrm{kV}$.

\section{Results}

In the hypodermal layer of the palmar side, the blood vessels are arranged in many different microvascular three-dimensional architectures that we can easily associate with the anatomical structures located there: the sweat glands, thermoreceptorial corpuscles and cutaneous sensory receptors, many glomic corpuscles and hair follicles.

We can describe the vascular supply of each of these systems starting from its three dimensional structures as seen by scanning electron microscopy.

\section{An overview}

Looking at the microvascular structure of the skin transversally sectioned, we can readily distinguish three vascular layers (Figure 1): one corresponding to the dermal papillae made up of digitiform spiral vessels, the vascular sheet of the dermal-epidermal junction and finally the microvascular specializations of the hypodermal layer. These are related to the corpuscles sited in this region and are represented by the sweat gland thermoreceptorial and tactile corpuscles, glomic bodies and hair follicles.

\section{Sweat glands}

In the human hand the sweat glands are mostly located in the palmar side of the skin, and are supplied by a dense capillary three-dimensional structure.

These vessels form a tubular-shaped structure around the gland faithfully reproducing the shape of both the gland itself and its excretory duct.

Looking at the three-dimensional microvascular structure of the gland we can distinguish two structures (Figure 2): the ovoid main body and the vessels supplying the excretory duct.

The main body has an ovoid shape made up of thin tortuous capillaries measuring about $7 \mu \mathrm{m}$ in diameter. At high magnification it is possible to see the characteristic tubular disposition of capillaries enveloping the tubules of the gland (Figure 3).

Sometimes it is possible to see two ovoid microvascular structures joined together with the same

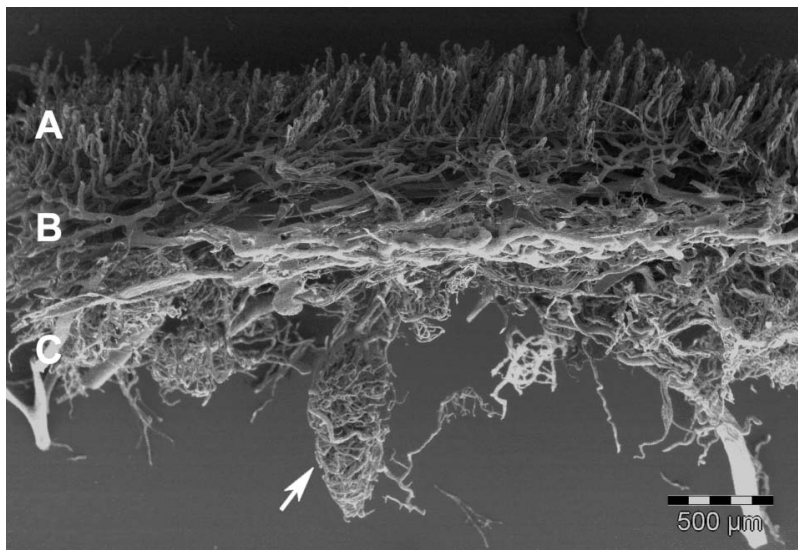

Figure 1. The microvascular structure of the transversally sectioned human digit (thumb): (A) papillary layer, (B) dermalepidermal junction, (C) hypodermal layer. Note the ovoid structure made up of the capillaries feeding a hair follicle (arrow). 


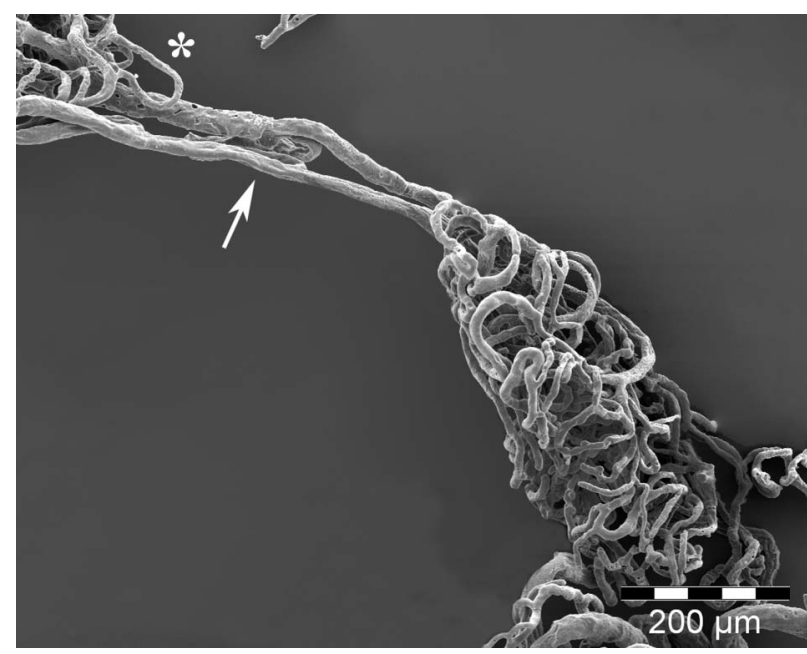

Figure 2. The microvascular structure of a sweat gland: note the afferent hypodermal arteriolar plexus $(*)$ feeding the excretory duct in the form of straight longitudinal capillaries (arrow), and the body of the gland by a thick ovoidal capillary plexus.

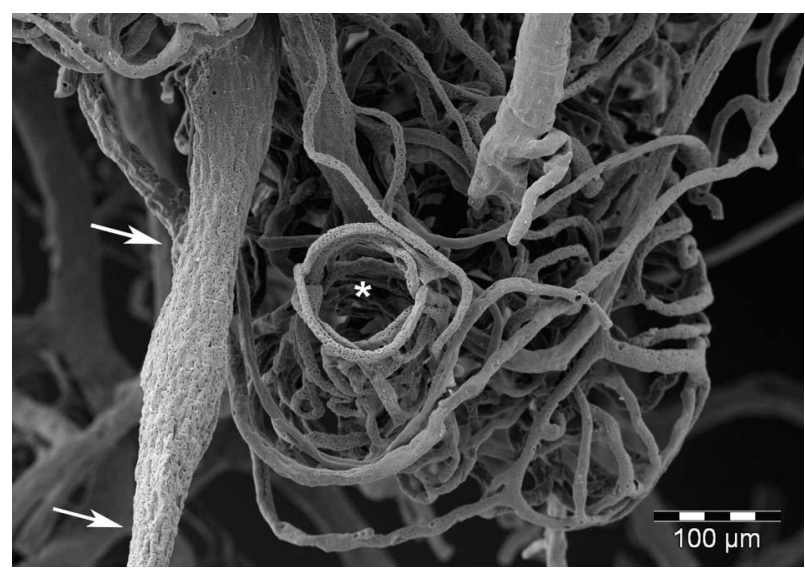

Figure 3. The capillaries feeding the excretory duct (*): a transverse view. Note the calibre changes of a medium sized arteriole (arrows).

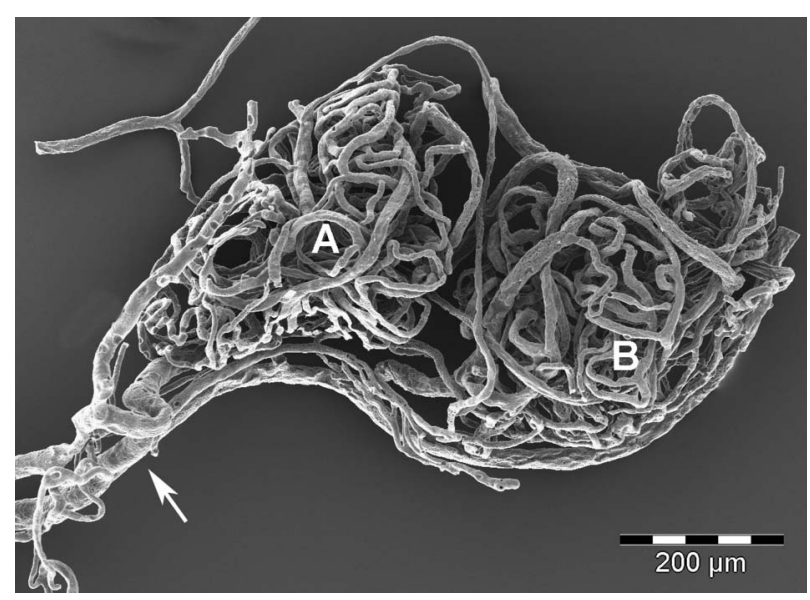

Figure 4. Sweat gland: two ovoid microvascular structures (A/B) joined together with the same vascular support. Note the unique excretory duct (arrow). vascular support. This is clearly visible when there is a single vascular tubule corresponding to the excretory duct (Figure 4).

The vascular structure of the sweat gland is large at the bottom of the gland then gradually decreasing up to the dermal-epidermal junction.

The excretory duct of a sweat gland can be variously supplied either by two or three row-like capillaries running parallel to the axis of the gland, or by spiral capillaries forming a tubular shaped vascular structure following that of the body of the gland itself.

\section{Thermoreceptorial and tactile corpuscles}

In the hypodermal layer we can readily identify other vascular structures visible as flake-like, cone shaped dense capillary bodies that may represent the vascular supply of thermoreceptorial and tactile receptors (Figure 5).

\section{Glomic bodies}

In the hypodermal layer we often find arteriovenous systems connected in series called glomic bodies (Figure 6): they are made up by an afferent arteriole giving origin to an interlaced capillary network ending in a venule.

In other cases glomeruloid bodies can be seen with a single tortuous arteriolar vessel ending in a venule or direct 'end to end' or 'end to side' connections.

We can easily distinguish the artery from the vein looking at the shallow impressions of various shapes and sizes left on the cast by the nuclei of endothelial cells: on arteries they are stretched and placed longitudinally along the major axis of the vessel, whereas in veins they are roundish and randomly placed on the cast's surface. Moreover, the shape of a transverse section of arterioles is circular while in veins we see an ovoid one (Figure 7).

\section{Hair follicles}

The vasculature of a hair follicle can be easily distinguished by its fusiform shape and a more dense capillary network with a greatly reduced intervascular distance.

Sometimes we can see the afferent vessels arising from the upper dermal-hypodermal longitudinal sheath and sometimes also directly from the hypodermal layer (Figure 8).

\section{Discussion}

The dermal layer of human skin contains many specialized vascular structures involved in supplying 


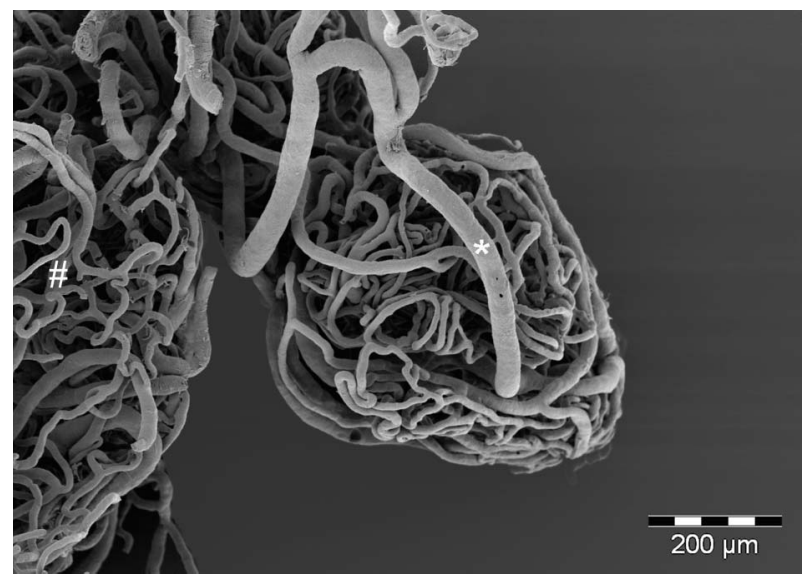

Figure 5. A thermoreceptorial or tactile corpuscle: note its conic shape that well fits the dermal papillae. An afferent artery is clearly visible on the vascular body of the corpuscle (*). Note the thick vascular capillary plexus of another thermoreceptorial corpuscle (\#).

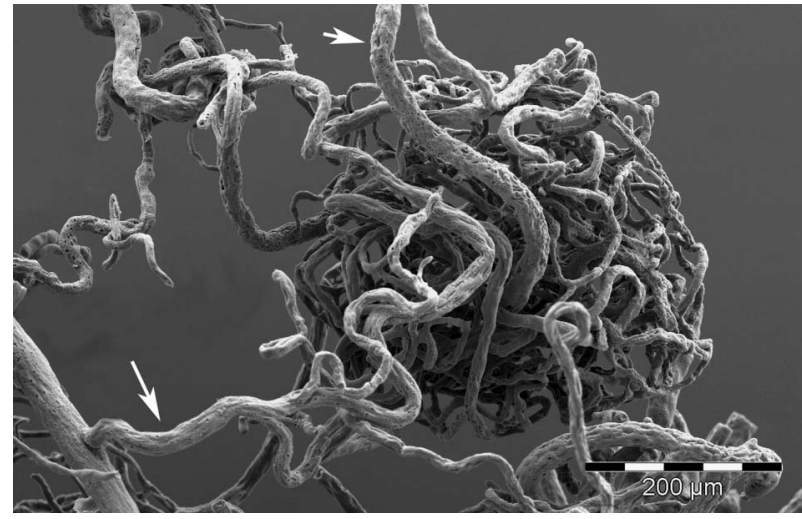

Figure 6. Glomic bodies in hypodermal layer: the characteristic 'glomeruloid' shape. Note the afferent artery (large arrow) and the efferent vein (small arrow).

excretory, mechanical, stereognosic and thermoregulatory functions like sweat glands, thermoreceptorial and tactile corpuscles, glomic bodies and hair follicles.

Corrosion casting combined with SEM analysis clearly depicted their vascular architecture focusing on their morphological features and three-dimensional disposition in space.

The sweat glands are clearly recognizable in the hypodermal layer of the skin thanks to their ovoid shape and the characteristic tubular vessels supplying the excretory duct well visible and always connected to the epidermal vascular plexus.

Sometimes two different ovoid vascular structures can be seen connected with a single excretory duct and supplied by the same vascular network: this may be due to the shape of the gland itself made up of two, three or more lobes.

This finding demonstrates the correspondence between the shape of the gland and its vascular support.
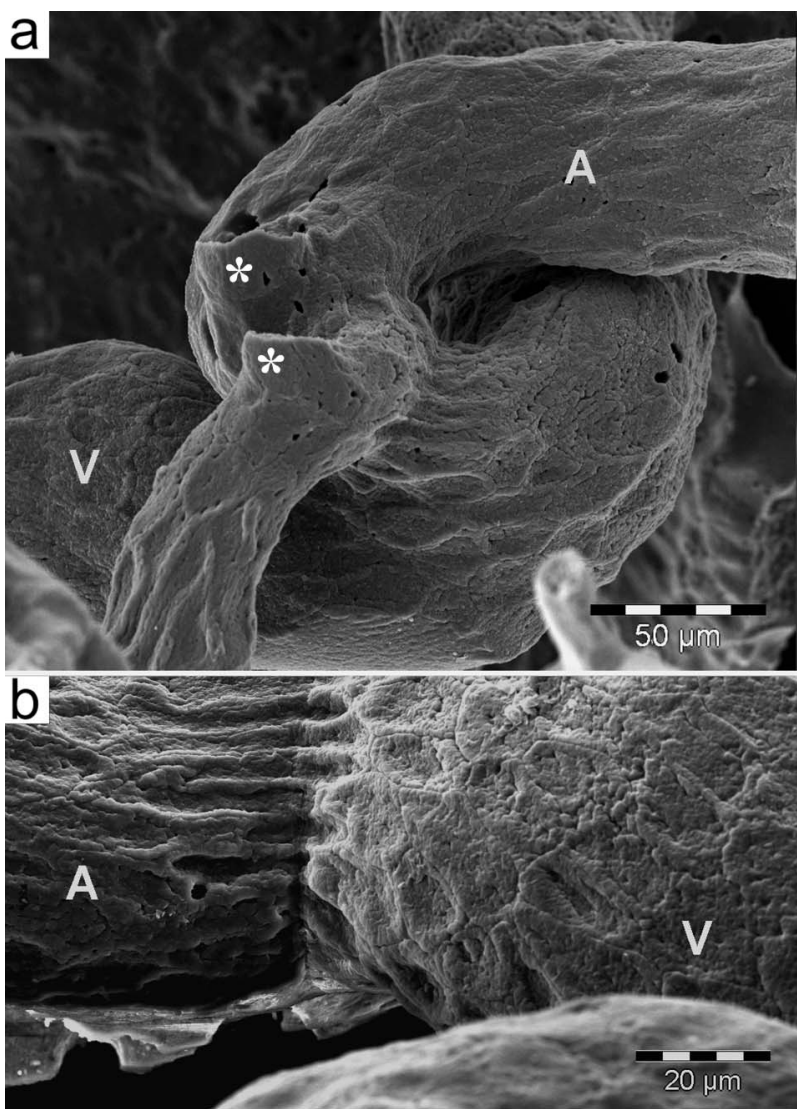

Figure 7. Arteriovenous connections: side-to-side (a) and end-toside anastomoses (b). Note the differences in endothelial cell imprints on the artery (A) and vein (V), and the broken vessels in (a).

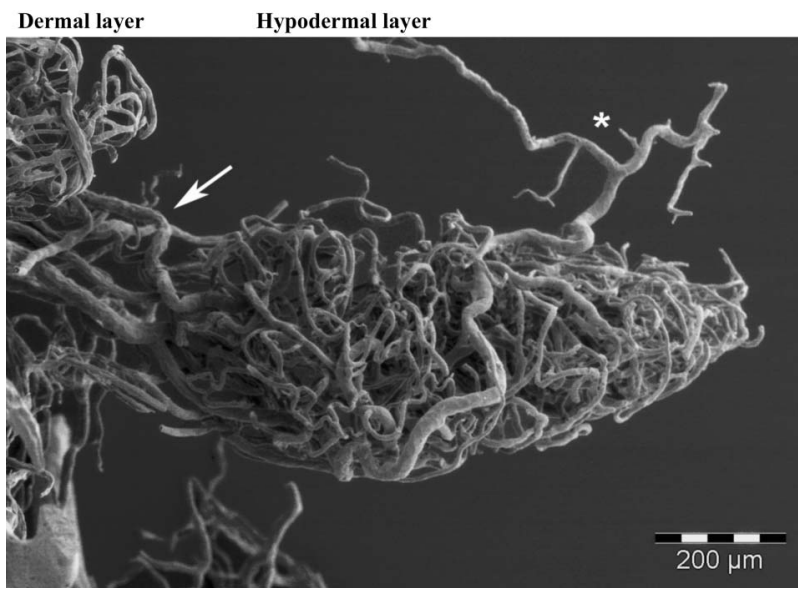

Figure 8. The microvascular structure of a hair follicle: note the arterioles arising from the dermal-hypodermal junction (arrow) and hypodermal layer $(*)$.

Other vascular structures are the capillaries supplying the hair follicles in the volar skin of the digit (Grunt et al. 1982). They are arranged in fusiform units directed perpendicular to the epidermal vascular sheath and well distinguishable from the sweat glands by the disposition of their capillaries: sweat 
gland capillaries are arranged to form an ovoid structure with a wide bottom gradually narrowing close to the dermal-hypodermal junction, whereas the hair follicle capillaries are fusiform.

We also analysed the microvascular structure of thermoreceptorial or tactile corpuscles even if is difficult to differentiate them because of their very similar vascular structure.

One feature that can help to distinguish these structures is their cone shape reflecting the shape of these corpuscles, often visible in dermal papillae.

We could also see some arteriovenous anastomoses sited mostly in the dermal layer: these shunting systems underlie many regulatory mechanisms regarding blood flow in the extremities. The impairment of these systems due to hereditary connectivitis, collagenopathies or chronic systemic disease (diabetes, atherosclerosis) may lead to a variety of pathological conditions. For example, a well known microangiopathy caused by diabetes and mostly affecting the extremities can cause an ischaemic impairment of the vascular systems feeding dermal corpuscles and cause both a loss of trophism and sensitivity of digital skin. Another syndrome affecting the capillaries of the extremities and whose pathogenesis is still not completely understood is Raynaud's phenomenon.

Knowledge of the normal morphological threedimensional structure of these systems will lead to a better understanding of the pathological mechanisms involved. If we focus on the morphological arrangements of capillaries in the hypodermal layer we can easily grasp how difficult it is to understand the regulatory mechanism in all the plexiform structures described. In addition, it is easy to see how an impairment in some of these vascular bodies could give rise to a number of microvascular diseases.

\section{References}

Backhouse KM. 1981. The blood supply of the arm and hand. In: Tubiana R, editor. The Hand. Philadelphia: WB Saunders. pp. 297-309.

Bryce LM, Chizuka IDE. 1988. The structure and function of cutaneous sensory receptors. Arch Histol Cytol 51(1):1-34.

Castenholz A. 1989. Interpretation of structural patterns appearing on corrosion casts of small blood and initial lymphatic vessels. Scanning Microsc 3(1):315-325.
Conrad MC. 1971. Role of the circulation of the skin in maintaining normal body temperature. In: Conrad MC, editor. Functional Anatomy of the Circulation to the Lower Extremities. Chicago: Year Book Medical Publishers. pp. 115-131.

De Takats G. 1932. Vascular anomalies of the extremities. Report of five cases. Surg Gynecol Obstet 55:227-237.

Edwards EA. 1960. Organization of the small arteries of the hand and digits. Am J Surg 99:837-846.

Grant RT, Bland EF. 1930. Observations on arteriovenous anastomoses in human skin and in the bird's foot with special reference to cold. Heart 15:385-411.

Grunt T, Lametschwandtner A, Staindl O. 1982. Die Angioarchitektur der Haut des retroaurikulären Bereiches des Menschen. Eine rasterelektronmikroskopische Untersuchung an Korrosionspräparaten. HNO 30:420-425.

Hodde KC, Nowell JA. 1980. SEM of micro-corrosion casts. Scan Electron Microsc (Pt 2):89-106.

Lametschwandtner A, Lametschwandtner U, Weiger T. 1990. Scanning electron microscopy of vascular corrosion casts technique and applications: Updated Review. Scanning Microsc 4(4):889-941.

Lametschwandtner A, Miodonski A, Simonsberger P. 1980. On the prevention of specimen charging in scanning electron microscopy of vascular corrosion casts by conductive bridges. Mikroskopie 36:270-273.

Murakami T. 1971. Application of the scanning electron microscope to the study of the fine distribution of blood vessels. Arch Histol Jpn 32(5):445-454.

Nasu T, Yamanaka T, Nakai M, Ogawa H. 1998. Scanning electron microscopic study of the vascular supply of the equine hoof. J Vet Med Sci 60(7):855-858.

Pollitt CC, Molyneux GS. 1990. A scanning electron microscopical study of the dermal microcirculation in the equine foot. Equine Vet J 22(2):79-87.

Roth SI. 1967. Hair and nail. In: Zelickson AS, editor. Ultrastructure of Normal and Abnormal Skin. Philadelphia: Lea and Febiger.

Sakita S, Ohtani O, Nakatani T, Morohashi M. 1994. Threedimensional microvasculature of the hair follicle. J Dermatol Sci 7(Suppl):S1-S4.

Straile WE. 1969. Vertical cutaneous organization. J Theor Biol 24(2):203-215.

Yamamoto O. 1990. Three-dimensional architecture of the microvasculature in the rat foot-pad, with special reference to vasculature around the eccrine sweat glands. A scanning electron-microscopic study of corrosion casts. Cell Tissue Res 262(2):225-232.

Weiger T, Lametschwandtner A, Stockmayer P. 1986. Technical parameters of plastics (Mercox CL-2B and various methylmethacrylates) used in scanning electron microscopy of vascular corrosion casts. Scan Electron Microsc (Pt 1):243-252. 